\title{
Sizing Function Based on Machine Learning for Unstructured Mesh Generation
}

\author{
Xiang Gao ${ }^{1,2}$, Chuanfu Xu' ${ }^{1,2}$ \\ ${ }^{1}$ College of Computer, National University of Defense Technology \\ Deya Road No.109, Changsha, China \\ gaoxiang12@nudt.edu.cn; xuchuanfu@ nudt.edu.cn \\ ${ }^{2}$ State Key Laboratory of High Performance Computing, National University of Defense Technology \\ Deya Road No.109, Changsha, China
}

\section{Extended Abstract}

In the past few decades, unstructured mesh generation has had great success. However, it is still a challenge work to prepare a suitable sizing function for the mesh generation process. A sizing function is used to define the distribution of element scales over the meshing domain, and a good one should define smaller scales in the region where geometrical and physical characteristics exist and larger scales elsewhere. Furthermore, to ensure the mesh quality in gradation regions so that the gradient of element scales must be limited in a reasonable range. Grid sources are effective ways to define sizing functions in many computational aerodynamics applications [1]. The time cost of defining sources may be affordable for simple configurations, but for complicated models, the interactive process that defines these sources is time-consuming and error-prone. Recently, an automatic sizing function defined at unstructured background mesh is proposed in [2,3]. This kind of algorithm starts from an initial mesh of the geometry model, in which initial element scales are defined by considering geometrical factors at mesh boundaries and several user-specified parameters. Then a convex nonlinear programming problem is formulated and solved to obtain a smoothed sizing function over this background mesh. Although the approach greatly reduces the labor time, it still needs to generate an initial unstructured mesh as the background mesh, and an interpolation step is required for places that not located at mesh nodes. In this work, we develop a novel sizing function based on machine learning methods like support vector machine [4] and deep neural network [5]. The proposed approach first distributes points on geometry surfaces and outer boundaries, and initial element scales of these points are defined adapted to curvatures and proximities. Then a machine learning model is trained by using the coordinates and scales of these points as training data. In addition, some special locations with expected scales could be add to the training data before training. In generation stage, every possible location of the meshing domain can input its coordinates into the model function, and a resulting element scale of this location will be given. Several simple cases are tested based on the NETGEN open source software [6], the results show that the proposed sizing function has good adaptability.

\section{References}

[1] R. Aubry, B. K. Karamete, E. Mestreau, S. Dey, R. Lohner, "Linear sources for mesh generation," SIAM J. Sci. Comput., vol. 35, no. 2, pp. A886-A907, 2013.

[2] J. Chen, Z. Liu, Y. Zheng, P. Zheng, J. Zheng, Z. Xiao, C. Yu, “Automatic Sizing Functions for 3D Unstructured Mesh Generation," Procedia Eng., vol. 203, pp. 245-257, 2017.

[3] J. Chen, Z. Xiao, Y. Zheng, J. Zheng, K. Liang, "Automatic sizing functions for unstructured surface mesh generation," Int. J. Num. Meth. Eng., vol. 109, no. 4, pp. 577-608, 2017.

[4] L. Wang, Support vector machines: theory and applications. Springer Science \& Business Media, 2005.

[5] J. Yosinski, J. Clune, Y. Bengio, H. Lipson "How transferable are features in deep neural networks?" Advances in neural information processing systems, pp. 3320-3328, 2014.

[6] J. Schoberl, "NETGEN: An advancing front 2D/3D-mesh generator based on abstract rules," Comput. Visualization Sci., vol. 1, pp.41-52, 1997. 